\title{
The Potentialities of the Expressive and Gestual
}

\section{Language}

\author{
Giuliana De Rosa ${ }^{1}$, Caterina Francone ${ }^{2}$, Guglielmo De Rosa ${ }^{2}$ and Patrizia Bruno ${ }^{3}$ \\ 1. Educator A.M.N.E.S.I.A. Villaricca, Corso Italia 395 Napoli Italia \\ 2. Psychologist-A.M.N.E.S.I.A. Villaricca, Corso Italia 395 Napoli Italia \\ 3. Geriatrician-UVA DS 35-37ASL NA2Nord Pozzuoli, Via Corrado Alvaro 7 Napoli Italia
}

\begin{abstract}
The aim of this work is to integrate the Educative intervention to Scientific research in support of the biological, psychological, social and economic discomfort of the Person affected by cognitive deficit. The specific objective is to stimulate the retained cognitive, emotive and motor abilities, educating to the theatricality through the establishment of a theatrical laboratory. Our experience was conducted with a group of elderly people affected by neuro-geriatric pathologies with medium-high cognitive decay. The encounters took place in the home of one of the members of the group. Fundamental was the actualisation of the process of individual and collective empowerment. They started with social activities and viewings of film scenes with Charlie Chaplin, actor to be observed, picked by the activities' participants. Finally, there was a re-enacting of a "wordless" scene through the reading of simple sentences to express in sign language. The parameters taken into consideration during the qualitative analysis are: attention, learning, expressivity and motor capabilities, measured at every encounter through a Likert scale of evaluation created ad hoc, directly proportional to the level of adequacy of the single ability. In conclusion, the maximum of scored points manifested itself in the attention and expressivity items.
\end{abstract}

Key words: Cognitive discomfort, communicability, theatre, emotions, mnemonic stimuli, empowerment.

\section{Introduction}

The group of elderly people that took part in the work described below was selected from the north of the city of Naples, in Italy, by the Centres for Cognitive Disorders and Dementias (CCDD) and the Association A.M.N.E.S.I.A. Italian acronym for Neurogeriatric Diseases and Alzheimer Syndrome Association. A.M.N.E.S.I.A. has amongst its main objectives, that to provide support to the familiar, informal and institutional network creating a system culture for taking care of the Neurogeriatric pathology. Amongst the activities that the A.M.N.E.S.I.A. team offers to people affected by Alzheimer, there are artistic laboratories such as the education to theatricality. The pedagogical value of the theatrical experience allows to reveal the active energies of the human expression and

Corresponding author: Patrizia Bruno, research field: dementia and Alzheimer's disease in the elderly population. to educate the elderly to creativity.

The Theatre-therapy combines the psychological-rehabilitative theories with the techniques and tools of art and acting. The diversification of interventions in such complex patients from a psycho-physical and social viewpoint has resulted in an excellent solution to favour the global rehabilitative intervention.

The experiences detected by our research, are those aimed at people with different types of pathologies of the affective sphere and neurogeriatric and they highlighted that the aim of theatre-therapy is to harmonise the relationship with one's self and with others through the body, the voice and gestures. The beneficial effects of group sittings produce results even outside of the scene, as the stimuli previously received become an integral part of people's lives.

For this reason we wanted to translate such objectives in the neurogeriatric field, trying to project 
an experience that unites psycho-physical needs of the elderly and our need to find strategies to improve the quality of life of each single individual, including families, a global care where the biological, physical, psychological and social aspects to consider, are analysed individually and developed in a single therapeutic path.

\section{Materials and Methods}

\subsection{Recruitment of Participants}

The group, very heterogeneous for levels of cognitive capabilities was formed of 15 elderly people selected from a nucleus of geriatric and psychological treatment that identified the elderly and the people to whom the activities in the project would be directed. This happened through:

- Analysis of the physical and psychological state of health of the patient through which information on the mental state and the grade of affectivity that characterises it is obtained. Such analysis of the medical case of the subjects was carried out through thorough medical-geriatric and neuropsychological valuations. The geriatric valuation designed to the definition of the medical case and of comorbidity through the multidimensional exam.

The formalised neuropsychological valuation was structured in a way to examine individually all cognitive (memory, language, attention, routine and executive functions) and affective domains. For the single psychometric tests used see Table 1.

- Analysis of the familiar sector in order to visualise all daily social relationships, the cultural level of the family and the relative possibilities of interaction by opening up to new prospectives of collaboration.

- Analysis of the environmental sector to evaluate the adequacy and security of the environment the elderly lives in.

The activities were carried out in a domestic environment, at the house of one of the group members willing to host. The logistical parameters for a correct reception were space, brightness of the environment and the minimum presence of architectural barriers.

The group of the 15 elderly was formed as follows:

- 5 with medium-grade cognitive deficit;

- 5 with light cognitive discomfort, but, with high motor disturbances;

- 2 with severe cognitive deficit;

- 3 patients diagnosed with depression and initial symptoms of cognitive impoverishment.

\subsection{Planning the Intervention}

The organisational asset of the therapeutic path of theatrical laboratory was structured and implemented as follows:

Table 1 Entry neuropsycholo-gical evaluation.

\begin{tabular}{|l|l|}
\hline $\begin{array}{l}\text { Italian Tests } \\
\text { calibration }\end{array}$ & Cognitive Function Explored \\
\hline Mini Mental State Examination (MMSE) & General cognitive functioning \\
\hline Geriatric Depression Scale (GDS) & Depression \\
\hline ADL & Daily life activity \\
\hline IADL & Daily life instrumental ability \\
\hline Trail Making test (Giovagnoli et al., 1996) & Sustained and divided attention \\
\hline Stroop Test: short version (Cafarra et al., 2002) & Inhibitory control and interference sensitivity \\
\hline Span di Cifre (Spinnler e Tognoni, 1987) & Short term verbal memory \\
\hline Span Spaziale- Test di Corsi (Spinnler e Tognoni, 1987) & Short term spatial memory \\
\hline Breve racconto(Spinnler e Tognoni, 1987) & Long term verbal memory \\
\hline Copia e rievocazione della Figura di Rey (Cafarra et al., 2002) & Long term spatial memory and visuospatial-visuocontructive abilities \\
\hline Fluenza Fonologica (Caltagirone et al., 1995) & Verbal fluency \\
\hline Clock Drawing test (Cafarra, 2011) & Visuospatial and visuocontructive abilities \\
\hline
\end{tabular}


- Identification of the specific objectives to reach: all aspects to stimulate that regard the "person" with cognitive discomfort were defined and, moreover, they were structured based on the pedagogical competences in play.

More precisely they were identified in:

Objectives to stimulate the Cognitive area:

Memory exercising

Education to the development of auditory and visual attention

Education to the logical thought and the formulation of answers

Objectives to stimulate the Motor area:

Finalised scenic movement and event consequentiality

Expressive and motor coordination

Involvement in rhythm and dance

Development of fine motor skills

Objectives to stimulate the emotive and relational sphere:

Development of the self-identity

Development of group identity

Acquisition of a "role" in the group

Development of self-esteem, creativity, enthusiasm

- Identification of the professional figures more apt

to carry out an activity path based on the pre-set objectives. They are:

Educators who have elaborated, when attributed the title, a thesis in Psychology of ageing

Musical therapists;

Art therapists;

Psychologists;

Physiotherapists.

The collaboration of voluntary musicians, beauticians, tailors, and young dancers completed the teamwork structure:

- Meeting and dialogue phase between participants

- Group motivation

- Structuring of a script to turn into a short theatrical representation.

- First hypothesis on the distribution of roles and the characters of the theatrical work

- Phase of induction to the recognition and of body and spatial-temporal identification

- Phase of recognition of one's own vitality

- Definitive attribution of roles and characters for the conclusive theatrical work of the project

- Personal phase of artistic and theatrical activity

- Conclusive phase represented by a final show

- Rating of results

- Conclusions

\subsection{Procedures}

The first two days of activity were dedicated to the meeting and dialogue phase between the participants. Each one told stories about their lives from which came out dreams, hopes, and nostalgias. From here came the need to show them the project in order to motivate them to a fun and recreational therapeutic path, highlighting the actuation of the process of individual and group Empowerment.

A recap of the identification of one's self and body through a puzzle of a human figure, previously divided in various pieces, including thoughts represented by clouds and cartoon bubbles, gave start to the successive phases.

In the following meetings the process started towards the achievement of the awareness that the soul and body are always "alive" and active when you can create, smile, cry and think, independently of your state of health. This happened through the game of "Positive thoughts" during which the elderly, holding each other's hands, had to race in the research of the biggest number of "nice thoughts" and "nice memories". It was useful to appreciate how much the competitive instinct favoured the research of long lost emotions and the discovery of a forgotten vitality.

From the fourth meeting onwards, it was possible to work, separately, on the writing of the script of the "comedy" to act out. This was elaborated putting together small parts of the stories of each member of the group and keeping into account the heterogeneity of 
the group from a mobility point of view as well. The vision of mute cinema scenes completed the space dedicated to these first phases of activity, to give start to the real theatrical laboratory. Charlie Chaplin was the actor identified by the elderly for them to observe his expressions, emotions, the contexts and everything that interested them. The continuous stimuli to reflection, as well as, auditory and visual, gave excellent results, making the ground to incentivise the work fertile.

From the fifth-sixth meeting to the end of the path, acting, simulation and interpretation of gestures and images exercises started. All exercises aimed at the learning of a new emotive vocabulary. The miming action between the participants was not simple to do and understand at the start but, through exercise and the exchange of dialogues to express without the use of words, excellent levels of expressivity and intuition were reached. Such training was possible giving, one by one, all members of the group small sheets to read, with, written on them, sentences or drawings to act out, just like "Charlie Chaplin", at the times of mute cinema. Rhythm and dances integrated the activity stimulating the elderly from an auditory and motor viewpoint, with the supervision of a Physiotherapist. The commitment and the objective to reach saw the exponential increase of everyone's participation even in the preparation of the costumes.

The fun, protagonist of every meeting, allowed a strong motivation of the group and favoured a bigger presence of family members, intrigued, attentive and more interested in the progress of their loved ones.

When it came to the final show titled: "Lights and shadows in a well of dreams", the above-mentioned professional figures joined in, with the task of valuing the show to represent from a scenic point of view. Therefore we were able to use the intervention of tailors, busy making costumes, beauticians who took care of the physical side, musicians and dancers who offered a spectacular choreography. Other volunteers, busy in all kinds of activities, made the representation even more interesting.

\section{Results}

This experience revealed that theatrical activity was able to grasp the aspects more useful to the socio-educative matter proposed as a general objective. Thus a significant goal reached was gaining the curiosity of the families, evermore present and attentive to the progress of their loved ones. This allowed the establishment of positive energies, and even the more distracted and more resistant of the elderly, felt less alone. Such conditions allowed the construction of positive collateral paths established in the families and in the contexts in which the participating elderly live. The relational difficulties between the families and the person affected by the neurogeriatric disease appeared diminished by the development of self esteem and of the identity of their loved ones.

The observation of the therapeutic progress of the artistic-theatrical path of the group of elderly was carried out evaluating 7 of the 15 meetings, i.e. every meeting in which more than $50 \%$ turned up.

The parameters taken into consideration during the qualitative analysis are: attention, learning, expressivity and motor capabilities, measured at every encounter through a Likert scale of evaluation created ad hoc, made up of ascendant scores from 0 to 4 , directly proportional to the level of adequacy of the single ability. The maximum of scored points manifested itself in the attention and expressivity items.

Table 2 reports the results of the group relative to the key items, derived from the mathematical average obtained from the sum of the individual scores of the elderly divided by the total number of people. For example: in the first meeting observed, the participants obtained the following scores for the key item relative to the level of attention:

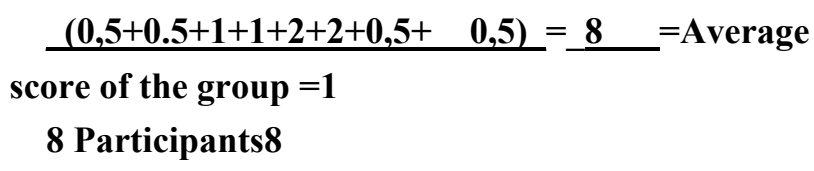

There were also positive elements that don't only regard the items taken into consideration but, also, those that concern memory. 
Table 2 Educative and occupational valuation of the group.

\begin{tabular}{llllllll}
\hline ITEM & 1st meeting & 2nd meeting & 3rd meeting & 4th meeting & 5th meeting & 6th meeting & 7th meeting \\
\hline Level of attention & 1 & 1.50 & 2 & 2 & 3 & 3.50 & 3.80 \\
Level of comprehension & 0.3 & 0.5 & 1 & 1 & 1 & 2 & 3 \\
Motor abilities & 0.3 & 0,5 & 2 & 2.5 & 2.5 & 3 & 4 \\
Level of expressivity & 2 & 2 & 3 & 4 & 4 & 4 & 4 \\
\hline
\end{tabular}

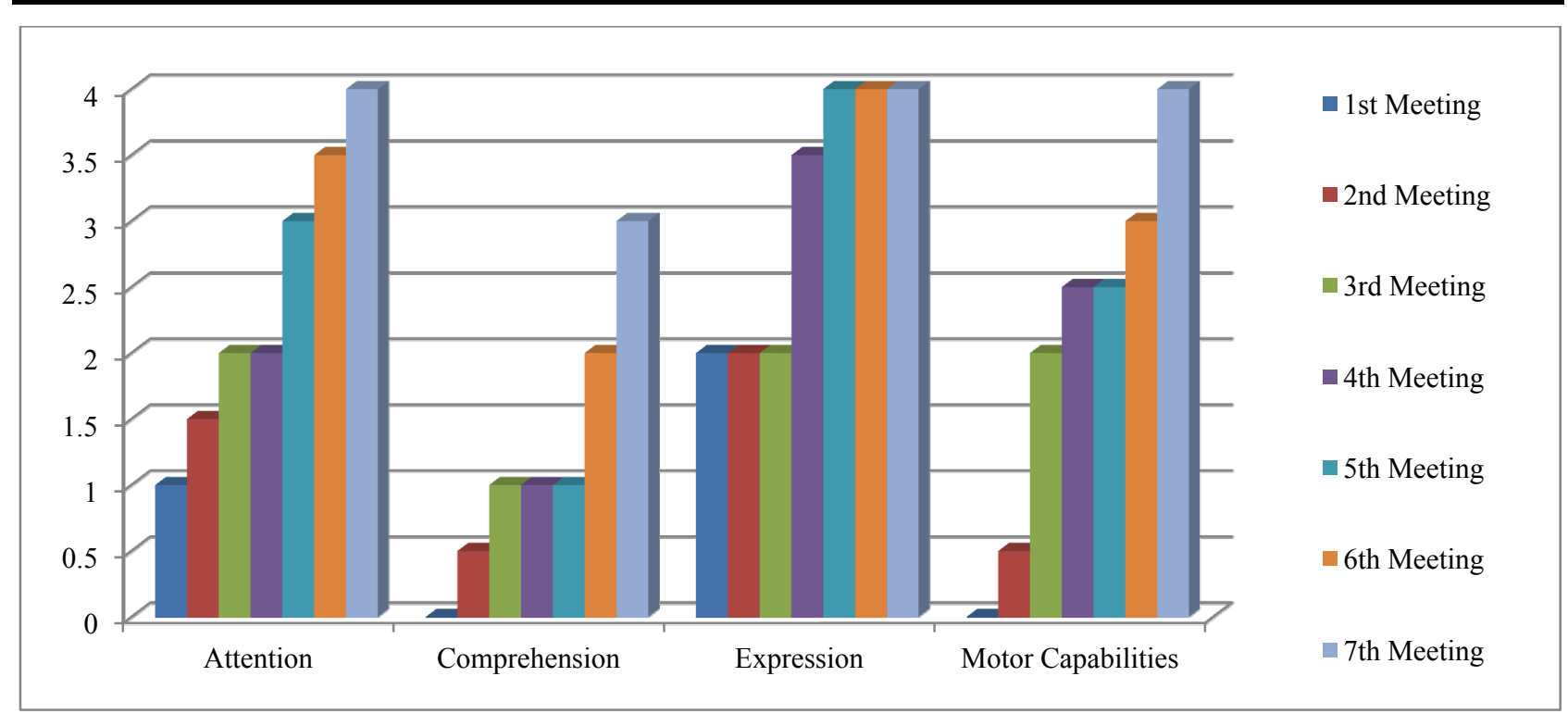

Fig. 1 Graphic representation of the results of the educative and occupational valuation of the group.

In fact, the memory not only of the plot but also of their own character, interiorised and enriched in the contents, remained alive and it was a subject of conversation between the participants even after the show. From our questions to the elderly on their experience it came up that, a big part of them, remembered the interpreted character, their lines, the spatial disposition of the structures and scenic objects and the colour of the costumes.

Even their auditory memory was highlighted after they listened back to the music used during the artistic performance. The sound stimuli helped them contextualise the event lived in the memory of the salient scenes.

\section{Conclusions}

The pedagogical research on therapeutic methodologies, aimed at elderly people with neurobiological damage and their families, has highlighted numerous works that treat the efficacy of "doing" theatre in support of a person with such discomforts.

It is necessary to research and favour alternative communication channels for elderly people affected by cognitive decay, in order to allow them to manifest their discomforts, fears, anxieties, emotions and pains. The stimulation to express themselves through acting, singing and gesticulating allowed notable progress for the wellbeing of the person.

The first observations highlight how Theatre-Therapy influenced some symptom logic aspects (significant mood and behavioural changes) and allowed the success of cognitive stimulation.

The experience confirms the initial hypothesis that demented subjects can hold onto information through highly motivational and emotional activity. These meetings show the therapeutic value in returning a sense of identity to the ill person, identity that tends to fade away with the incessant advancement of the disease. 
Counting on the reacquisition of a sense of "self" as a person that can still "do", with the due strategies, leads to a development of the conserved abilities. The improvement of mood, the increase in motivation to do, the expression of their own emotive world through the stimulation of creativity represent the added values and, at the same time, the objectives that every global care, to be truly called therapeutic, should have the quantification of the results themselves, especially in the specific areas of pedagogical and neuropsychological competence, such as memory, relational capabilities, behavioural disturbances and re-entry in the family, is and will always be our aim.

\section{References}

[1] Boella, L., and Buttarelli, A. 2000. Per amore di altro, Milano, Raffaello Cortina Editore.

[2] Braun, M. 1994. Ginnastica Espressiva, I movimenti per chi è nell'età d'argento della vita, Como, red edizioni.

[3] Bruner, J. 1997. La cultura dell'educazione. Nuovi orizzonti per la scuola, Milano, Feltrinelli.

[4] Bruscia, K. E. 1998. Defining music therapy, Barcellona, Gilsum.

[5] Caffarra, P., Vezzadini, G., Dieci, F., Zonato, F., and Venneri, A. 2002. "Rey-Osterriethcomplex Figure: Normative Values in an Italianpopulation Sample." Neurol Sci. 22: 443-7.

[6] Caffarra, P., Vezzadini, G., Dieci, F., Zonato, F., and Venneri, A. 2002. "Una versione abbreviata del test di Stroop: dati normativi nella popolazione italiana." Rivista di Neurologia 12 (4).

[7] Caffarra, P., Gardini, S., Zonato, F., Concari, L., Dieci, F., Copelli, S., Freedman, M., Stracciari, A., and Venneri, A. J. 2011. "Italiannorms for the Freedmanversion of the Clock DrawingTest.” Clin. Exp. Neuropsy Chol. Nov. 33 (9): 982-8.

[8] Caltagirone, C., Gainotti, G., and Carlesimo, G. A., et al. 1995. "Batteria per la valutazione del Deterioramento Mentale (parte 1): descrizione di uno strument di diagnosi neuropsicologica: Archivio di Psicologia." Neurologia e Psichiatria 4: 461-70.

[9] Castello, R. P. 2008. Festa di note, conoscere la musica, Milano, Mondadori.

[10] Cesa-Bianchi, M., and Cristini, C. 2009. Vecchio sarà lei!, Muoversi, pensare, comunicare, Napoli, Guida.

[11] Cristini, C., and Porro, A., 2008. Medicina e Musica,
Radiano (Bs), GAM.

[12] De Marinis M. 1988. Capire il teatro, La casa Usher, Firenze.

[13] De Michele, R., and De Rosa, E. 2009. Fortissimo, La musica dei bambini. Ricerca e intervento sperimentale sull'applicazione della musicoterapia in ambito scolastico, Roma, Ismez.

[14] Demetrio, D. 2000. L'educazione interiore, La Nuova Italia, Firenze.

[15] Demetrio, D. 1997. Manuale di educazione degli adulti, Laterza, Bari.

[16] Dewey, J. 1961. Come Pensiamo, La Nuova Italia, Firenze.

[17] Dewey, J. 1982. Il mio credo pedagogico, Antologia di scritti sull'educazione, La nuova Italia, Firenze.

[18] Diderot, D. 1989. Paradosso sull'attore, Editori Riuniti, Roma.

[19] Duccio Demetrio, 2013, L'autobiografia come cura di se. Cortina Raffaello (collana Mini). D'Amico S. Storia del teatro drammatico, Milano, Garzanti, 1937 (ed. rid. a cura di A. D'Amico, Roma, Bulzoni 1982).

[20] Giovagnoli, A. R., Del Pesce, M., Mascheroni, S., Simoncelli, M., Laiacona, M., and Capitani, E. 1996. "Trailmaking Test: Normative Values from 287 Normal Adult Controls." Ital. J. Neurol. Sci. 17 (4): 305-9.

[21] Katz, T. F. 1963. "Indice di dipendenza nelle attività della vita quotidiana (scala ADL). A.D.L. Activities of Daily Living." JAMA 185: 914.

[22] Lawton, M. P. 1969. "Indice di dipendenza nelle attività strumentali della vita quotidiana (scala IADL) InstrumentalActivities of Daily Living." Gerontologist 9: 179.

[23] Marinacci, S. 2010. Relazione Work Schop terapia artistica, Napoli, Associazione Vox Alterna.

[24] Measso, G., Cavarzeran, F., Zappalà, G., Lebowitz, Barry, D., Crook, Thomas, H., Pirozzolo, Francis, J., Amaducci, Luigi, A., Massari, D., and Grigoletto, F. 1993. "The Mini-mental State Examination: Normative Study of an Italian Random Sample.” Developmental Neuropsychology 9 (2): 77-85.

[25] Orefice, P., Sarracino, V., Guetta, S., Iavarone, M. L., Schettini, B., and Striano, M. 2008. Nuove questioni di pedagogia sociale, Milano, Franco Angeli.

[26] Sheikh, J. I., and Yesavage, J. A. 1986. "Geriatric Depression Scale (GDS): Recent Evidence and Development of a Shorter Version." Clinical Gerontologist 5: 165-73.

[27] Spinnler, M., and Tognoni, G. 1987. "Standardizzazione e taratura italiana di test neuropsicologici." The Italian Journal of Neurological Science 8 (6). 